\title{
Drug Effects on Newborn
}

1. In general, newborns are more sensitive than adults to the depressant effects of drugs

2. The seizure threshold is similar in newborns and adults

3. Newborns have larger volumes of distribution, requiring a higher dose of local anesthetic for toxic effects compared to adults

4. Changes in fetal heart rate after administration of local anesthetics are most often due to indirect effects (e.g. maternal hypotension and uterine hyperstimulation)

5. Preterm infant is more vulnerable than term infant to effects of analgesic and anesthetic drugs due to:

(a) Less protein available for drug binding

(b) Higher levels of bilirubin present and competing with drugs for protein binding

(c) Greater access of drugs to the CNS due to poorly developed blood-brain barrier

(d) Preterm infant has greater total body water and less fat content

(e) Preterm infant has diminished ability to metabolize and excrete drugs

6. Neuraxial opioids have a favorable effect on neonatal outcome compared to systemic opioid

(a) Better Apgar scores, and umbilical cord blood gas and $\mathrm{pH}$ measurements at delivery with neuraxial opioids compared to systemic opioids

(b) Maternal epidural opioid administration by continuous infusion rarely results in drug accumulation and subsequent neonatal depression

(c) Possible direct effect on the neonate at delivery due to systemic absorption and indirect effects by opioid-related maternal side effects (i.e., respiratory depression and hypoxemia) 